CARTA AL EDITOR

\section{DETERMINACIÓN DE RIFAMPICINA EN PLASMA HUMANO MEDIANTE UN MÉTODO DE CROMATOGRAFÍA LÍQUIDA}

\section{DETERMINATION OF RIFAMPIN IN HUMAN PLASMA BY A LIQUID CHROMATOGRAPHY METHOD}

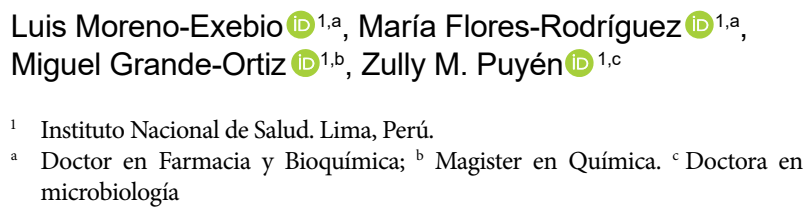

Sr. Editor: El desarrollo o la optimización de métodos para determinar las concentraciones plasmáticas de las principales drogas utilizadas en el tratamiento de la tuberculosis (TB) es fundamental para hacer un seguimiento a los pacientes y evaluar su adherencia al tratamiento. El monitoreo terapéutico de drogas (MTD) es el proceso de obtención de las concentraciones séricas de un fármaco y posterior modificación de la dosis basada en los resultados, optimizando los beneficios terapéuticos en estudios clínicos, mientras se reducen sus riesgos por efectos adversos o toxicidad $^{(1)}$. Actualmente, el MTD no es de aplicación regular en el Perú; sin embargo, el Instituto Nacional de Salud del Perú viene evaluando su utilidad a través de un estudio de adherencia al tratamiento anti-tuberculosis.

La rifampicina es parte fundamental del núcleo terapéutico en los regímenes anti-tuberculosis en el Perú y en el mundo ${ }^{(2)}$. En este sentido, es necesario evaluar la concentración sérica de rifampicina en pacientes que la reciben dentro de su tratamiento, para lo cual hay diferentes métodos rápidos y simples basados en cromatografía líquida de alta resolución; sin embargo, la mayoría de estos demandan procedimientos de preparación de muestras tediosos o requieren equipamiento especial. La utilización de cromatografía líquida para realizar el MTD en pacientes con esquemas de tratamiento de primera línea podría evitar o disminuir el uso de agentes de segunda línea, que son de alto costo y que demandan buena parte del presupuesto del Ministerio de Salud asignado al programa de TB.

Citar como: Moreno-Exebio L, Flores-Rodríguez M, Grande-Ortiz M, Puyén ZM. Determinación de rifampicina en plasma humano mediante un método de cromatografía líquida. Rev Peru Med Exp Salud Publica. 2020;37(2):385-6. doi: https://doi.org/10.17843/rpmesp.2020.372.5058

Correspondencia: Luis Enrique Moreno Exebio, lemoreno70@hotmail.com

Recibido: 20/12/2019 Aprobado: 15/04/2020 En línea: 12/06/2020
El presente estudio describe una nueva metodología rápida, con mejor selectividad para la determinación de la concentración de rifampicina en plasma humano, basada en el método desarrollado por Moreno y Grande ${ }^{(3)}$. Dicho estudio arroja resultados adecuados y correctos; sin embargo, tiene el inconveniente de tener una corrida cromatográfica larga y que el estándar interno utilizado al ser la rifampicina quinona, que tiene una estructura química similar a la rifampicina, podría interferir con la cuantificación de la rifampicina. Por lo descrito, se ha realizado el cambio del estándar interno (papaverina clorhidrato por rifampicina quinona) y el tipo de columna cromatográfica utilizada: C18 (XTerra $\left.{ }^{\circledR}\right) 150$ X 4,6 mm ID, $5 \mu \mathrm{m}$ tamaño de partícula (Waters, Irlanda), los cuales constituyen los principales cambios realizados al método anteriormente publicado ${ }^{(3)}$. Un método de cromatografía líquida más rápido, lo hace más económico porque consume menor cantidad de insumos y permite analizar un número mayor de muestras.

La validación del método bioanalítico se realizó de acuerdo con la guía correspondiente emitida por la Agencia de Medicamentos y Alimentación (FDA, por sus siglas en inglés) ${ }^{(4)}$, tomándose en cuenta la selectividad, la curva de calibración, la precisión, la exactitud, la recuperación y la estabilidad. En cuanto a la selectividad, las soluciones estándares de isoniazida, pirazinamida y etambutol no presentan señal que pueda interferir con el tiempo de retención de la rifampicina (4,5 $\mathrm{min})$ y papaverina clorhidrato $(2,6 \mathrm{~min})$, utilizado como estándar interno. La curva de calibración se obtuvo por regresión lineal $\left(\mathrm{r}^{2}=0,99\right)$, en el rango de 0,5 $250 \mathrm{ug} / \mathrm{mL}$. La precisión fue evaluada por la relación del área del pico de rifampicina sobre el estándar interno en cinco inyecciones sucesivas de los estándares de control de calidad. La precisión del método fue evaluada mediante el coeficiente de variación de cada concentración y la exactitud con el porcentaje; en ambos casos no debe superar el 20\% para el límite más bajo de cuantificación y el 15\% para los estándares de control de calidad. Los valores resultantes de límite más bajo de cuantificación y de estándares de control de calidad se presentan en la Tabla 1.

La recuperación promedio $(n=5)$ alcanzada con este método fue óptima, con un valor de $82,9 \%$. Sobre la estabilidad de las muestras a corto plazo, no se observó degradación en las muestras descongeladas en 0 y $24 \mathrm{~h}$. La respuesta para rifampicina en plasma, medida como la razón del área del pico (rifampicina versus el estándar interno) en las muestras preparadas y analizadas después de $24 \mathrm{~h}$, fueron $97,1 \%$ y $97,8 \%$ comparadas con el tiempo $t=0$ a las concentraciones de 5 y $100 \mu \mathrm{g} / \mathrm{mL}$, respectivamente.

La validación del método se realizó con 18 muestras de pacientes almacenadas en el laboratorio, las concentraciones fluctuaron entre 0,5 y $13,7 \mu \mathrm{g} / \mathrm{mL}$, valores que estuvieron dentro de la curva de calibración. 
Tabla 1. Límite de cuantificación, precisión y exactitud del método de cromatografía líquida de alta resolución para determinar la rifampicina en plasma humano

\begin{tabular}{|c|c|c|c|c|c|c|}
\hline \multirow[b]{2}{*}{ Parámetro $(\mu \mathrm{g} / \mathrm{mL})$} & \multicolumn{3}{|c|}{ Precisión } & \multicolumn{3}{|c|}{ Exactitud } \\
\hline & Promedio & $\begin{array}{l}\text { Desviación } \\
\text { estándar }\end{array}$ & $\begin{array}{l}\text { Coeficiente de } \\
\text { variación (\%) }\end{array}$ & $\begin{array}{c}\text { Concentración } \\
\text { nominal } \\
(\mu \mathrm{g} / \mathrm{mL}) \\
\end{array}$ & $\begin{array}{c}\text { Concentración } \\
\text { obtenida } \\
(\mu \mathrm{g} / \mathrm{mL}) \\
\end{array}$ & Exactitud (\%) \\
\hline $\begin{array}{l}\text { Límite más bajo de } \\
\text { cuantificación }\end{array}$ & 0,5 & 0,0 & 3,3 & 0,5 & 0,6 & 16,3 \\
\hline $\begin{array}{l}\text { Control de calidad A } \\
\text { (concentración más baja) }\end{array}$ & 5,2 & 0,0 & 0,7 & 5,2 & 5,8 & 13,0 \\
\hline $\begin{array}{l}\text { Control de calidad B } \\
\text { (concentración intermedia) }\end{array}$ & 20,6 & 0,0 & 0,5 & 20,6 & 21,3 & 3,4 \\
\hline $\begin{array}{l}\text { Control de calidad C } \\
\text { (concentración más alta) }\end{array}$ & 103,1 & 0,0 & 0,8 & 103,1 & 97,3 & $-5,7$ \\
\hline
\end{tabular}

En conclusión, el método propuesto, además de ser preciso y exacto, es más rápido, con una duración total en la corrida de 7 vs. 16 minutos, en relación con el método anteriormente publicado y realizado en el Instituto Nacional de Salud del Perú ${ }^{(3)}$. Esto significa una reducción del tiempo del $114 \%$ y constituye una buena alternativa para pruebas de monitoreo terapéutico de rifampicina, especialmente en lo que respecta a adherencia al tratamiento de pacientes con tuberculosis, que continúa siendo una de las principales barreras para el éxito del tratamiento.

Contribución de autoría: LME, MFR, MGO, ZPG han participado en la idea de investigación, concepción del artículo, recolección de datos, material de estudio y redacción del artículo. Todos los autores aprobaron la versión final.
Conflictos de interés: Los autores declaran no tener conflicto de interés en la publicación de este artículo.

Fuente de financiamiento: Autofinanciado.

\section{REFERENCIAS BIBLIOGRÁFICAS}

1. Peloquin C. Therapeutic Drug Monitoring in the treatment of tuberculosis. Drugs 2002; 62(15):2169-2183.

2. Ministerio de Salud Perú. Norma Técnica de Salud para el Control de la Tuberculosis. NTS. No 041-MINSA/DGSP - V.01.2006. Disponible en: http:// www.tuberculosis.minsa.gob.pe/portaldpctb/Contenido.aspx?op=1007.

3. Moreno-Exebio L, Grande-Ortiz M. Validación de un método de cromatografía líquida para la determinación de rifampicina en plasma humano. Rev Peru Med Exp Salud Pública 201431(1):56-61.

4. Center for Drug Evaluation and Research (CDER) FDA. Guidance on Bioanalytical Method Validation. May 2001. Disponible en: https://www. fda.gov/files/drugs/published/Bioanalytical-Method-Validation-Guidance-for-Industry.pdf 